Supporting Information

\title{
Sunlight Converts Polystyrene to Carbon Dioxide and Dissolved Organic Carbon
}

Authors: Collin P. Ward ${ }^{1 *}$, Cassia J. Armstrong ${ }^{1}$, Anna N. Walsh ${ }^{1,2}$, Julia H. Jackson ${ }^{1}$, and Christopher M. Reddy ${ }^{1}$

\section{Affiliations:}

${ }^{1}$ Department of Marine Chemistry and Geochemistry, Woods Hole Oceanographic Institution, Woods Hole, Massachusetts 02543, USA.

${ }^{2}$ Department of Civil and Environmental Engineering, Massachusetts Institute of Technology, Cambridge, Massachusetts 02139, USA.

\section{*Correspondence to:}

Collin P. Ward

Department of Marine Chemistry and Geochemistry

Woods Hole Oceanographic Institution

Woods Hole, MA 02543

Tel: 508-289-2931

Email: cward@whoi.edu 


\section{Materials and Methods}

\subsection{Polystyrene Sources}

Five polystyrene (PS) sources were used in this study: Goodfellow, Sigma 35K, Sigma 192K, Trycite 8001 , and Trycite 8003 . They are commercially available and vary in their physical and chemical properties. Goodfellow PS is a $196 \pm 1 \mu$ m-thick $( \pm 1 \mathrm{SE}, \mathrm{N}=3)$, clear, biaxially oriented film that has previously been used as a pure PS source in photochemical studies. ${ }^{1}$ Sigma $35 \mathrm{~K}$ and Sigma $192 \mathrm{~K}$ are PS pellets sourced from Sigma Aldrich, with polymer sizes of 35,000 and $192,000 \mathrm{Da}$, respectively. The $192 \mathrm{~K}$ pellets were spherical in shape with an average diameter of $3.5 \pm 0.1 \mathrm{~mm}( \pm 1 \mathrm{SE}, \mathrm{N}=10)$. The $35 \mathrm{~K}$ pellets were teardrop in shape with an average diameter of $2.4 \pm<0.1 \mathrm{~mm}( \pm 1 \mathrm{SE}$, $\mathrm{N}=10$ ). Both are sold as pure sources of PS. Trycite 8001 and 8003 were obtained from Transcendia, Inc., an international manufacturer, converter, and distributer of plastic films (www.transcendia.com). Trycite 8001 is a $44 \pm 1 \mu \mathrm{m}$-thick ( $\pm 1 \mathrm{SE}, \mathrm{N}=3$ ), clear, high-gloss,

low orientation pure PS film. Trycite is a $56 \pm 1 \mu \mathrm{m}$-thick ( $\pm 1 \mathrm{SE}, \mathrm{N}=3$ ), clear, low-gloss, 
low orientation film with black-rubber particle additives to yield a rigid, high-impact product.

\subsection{Light Absorption Properties}

Ultraviolet and visible light absorbance was measured using a Perkin Elmer Lambda 650s

equipped with a 150-mm integrating sphere. PS films (Goodfellow, Trycite 8001 and 8003) were cut into $2.5 \times 2.5 \mathrm{~cm}$ squares and attached to a clip-style center-mount accessory for absorbance measurement. Pelletized PS sources (Sigma 35K and 192K) were dissolved in chromatography-grade methylene chloride, transferred to a 1-cm cuvette, and analyzed on the spectrophotometer. Naperian absorption coefficients were calculated by multiplying absorbance $(\mathrm{A})$ by the dilution faction (where applicable) and 2.303 , and then dividing by the film thickness or pathlength of the quartz cuvette $(\mathrm{m})$. Film thickness was measured using a NIST-calibrated Mitutoyo micrometer with precision of 1 micron.

\subsection{Complete and Partial Photochemical Oxidation}


Complete and partial photochemical oxidation of PS was quantified following previously described approaches. ${ }^{2-4}$ Polystyrene films $(8 \times 1.5 \mathrm{~cm}$; Goodfellow $=0.25 \mathrm{~g}$, Trycite 8003 $=0.06 \mathrm{~g}$, Trycite $8001=0.05 \mathrm{~g})$ or pellets $(0.75 \mathrm{~g}$ per tube) were placed into air-tight, 12 $\mathrm{mL}$ quartz vials. For Trycite 8001 , the thinnest of all PS sources (Table S1), four films were stacked prior to placement in the quartz vials to increase pathlength and thus the rate of light absorption. The pre-combusted vials were filled with no headspace and sealed with GL-14 caps with custom-made 3-mm thick Viton septa. Laboratory atmosphere equilibrated synthetic seawater ( $35 \mathrm{~g} \mathrm{~L}^{-1}$ sodium chloride) adjusted to $\mathrm{pH} 8.2$ \pm 0.1 with sodium hydroxide and poisoned with saturated mercuric chloride $(0.1 \%$ saturated $\mathrm{HgCl}_{2}$ by volume) was used in all experiments. The vials were exposed to simulated sunlight emitted by an Atlas XLS+ solar simulator equipped with a long-arc Xe lamp and a Daylight filter (Ametek Inc.). UV-B and UV-A transmitting acrylic (Arkema Inc.) served as a thermal barrier between the light source and the quartz vials by absorbing infrared light. The vials were placed on cold plates (Aavid Thermaolly) equipped to a water-circulating bath to maintain sample temperatures at $25^{\circ} \mathrm{C}$ or $35^{\circ} \mathrm{C}$. Dark-controls were wrapped in aluminum foil and run alongside light-exposed treatments. Simulated 
sunlight exposure times were chosen to yield robust production of $\mathrm{CO}_{2}$ without limiting oxygen availability. Exposure times varied from six to 48 hours. Following a similar protocol as described above, but in a separate experiment, Goodfellow PS was also subjected to multiple exposure times from 12 to 72 hours. Following irradiation, photochemical $\mathrm{CO}_{2}$ production and $\mathrm{O}_{2}$ consumption were quantified. $\mathrm{CO}_{2}$ production was quantified $(\mu \mathrm{M})$ as the light minus dark difference in dissolved inorganic carbon (AS-C3 DIC analyzer; Apollo SciTech, Inc.). Oxygen consumption was quantified $(\mu \mathrm{M})$ as the dark minus light difference in dissolved oxygen using membrane inlet mass spectrometry (Bay Instruments, Inc.). Photo-vials containing only synthetic seawater (i.e., no PS) were exposed for 72 hours and neither $\mathrm{CO}_{2}$ production nor $\mathrm{O}_{2}$ consumption was detected. Furthermore, neither $\mathrm{CO}_{2}$ production nor $\mathrm{O}_{2}$ consumption was detected in dark controls. Four replicates were analyzed for photochemical $\mathrm{CO}_{2}$ production and $\mathrm{O}_{2}$ consumption.

To assess the production of dissolved organic carbon, $25 \mathrm{~cm}^{2}$ disks of Goodfellow PS were place in $300 \mathrm{~mL}$ beaker containing $70 \mathrm{~mL}$ of poisoned synthetic seawater. The beakers were covered with quartz lids, placed in a $25^{\circ} \mathrm{C}$ water bath held in the solar 
simulator, and irradiated for up to five days. Dark controls were wrapped in aluminum foil and ran alongside light-exposed treatments. At set time points, the beakers were removed from the simulator. Evaporation during the experiment was assessed volumetrically and never exceeded $10 \%$ of the initial sample volume. All samples were diluted back to the initial volume using Milli-Q water prior to analysis. Following dilution, the water was passed through a pre-combusted GF/F filter (nominal $0.7 \mu \mathrm{m}$ pore-size, Whatman) and acidified with trace-metal grade $\mathrm{HCl}$. Duplicate measurements of DOC were conducted using a Shimadzu 5000A TOC analyzer. ${ }^{5}$

Incident irradiance (W m $\left.\mathrm{m}^{-2} \mathrm{~d}^{-1} \mathrm{~nm}^{-1}\right)$ was measured using a NIST-calibrated spectroradiometer (StellarNet Black Comet C-50). This approach has previously compared favorably to irradiance determined using chemical actinometry. ${ }^{2}$ The samples were oriented horizontally in the center of the simulator along the profile of the long-arc lamp, which minimized irradiance variability to $<5 \%$ across all sample positions. Daily simulated UV irradiance (W m ${ }^{-2} \mathrm{~d}^{-1}$ integrated from $280-400 \mathrm{~nm}$ ) was compared to natural sunlight from 0 to $50^{\circ} \mathrm{N}$ across all four seasons ${ }^{6}$ (Table S1 and S2, Fig. S1). These 
latitudes were chosen because they encompass the mouths of the ten rivers that are currently estimated to export $90 \%$ of plastic waste to the oceans. ${ }^{7}$ The ratio of simulated to natural sunlight ranges from 3 to 24 , indicating that 1 day of simulated sunlight is equivalent to 3 to 24 days of natural sunlight. On average, the ratio is $5 \pm 4( \pm 1 \mathrm{SD}, N=24)$.

\subsection{Lifetimes of Complete and Partial Photo-oxidation}

Lifetimes of PS complete and partial photo-oxidation were calculated for Goodfellow and Trycite 8003 (Table S5). These PS samples were chosen because the experimental conditions were far simpler than the three other samples (i.e., individual films vs. stacked films or pellets). The mass of PS that was completely oxidized was calculated by multiplying $\mathrm{CO}_{2}$ production (Table $\mathrm{S} 1$ ) by the volume of the $12 \mathrm{~mL}$ photo-vials. The mass of PS that was partially oxidized was calculated similarly, but assuming that one mol of $\mathrm{CO}_{2}$ is required to produce one mol of $\mathrm{O}_{2}$. The excess oxygen was assumed to be incorporated at an oxygen to carbon $(\mathrm{O} / \mathrm{C})$ stoichiometry of 0.5 . This approach likely underestimates partial photo-oxidation because less than one mol is often required to

produce one mol of $\mathrm{CO}_{2}$, and the $\mathrm{O} / \mathrm{C}$ of partial transformation products is likely $<0.5{ }^{8,9}$ 
Assuming first-order kinetics, when plotting the natural log of the initial mass of PS and the mass of PS completely or partial photo-oxidized versus exposure time to simulated sunlight, the slope is equal to the rate of complete and partial photo-oxidation $\left(\mathrm{k} ; \mathrm{hr}^{-1}\right)$. By dividing the natural log of two by the rate, half-lives were calculated. The half-lives determined for simulated irradiance were converted to natural irradiance using the factors presented in Table S2. As described in the main text, these early estimates may vary depending on several factors that are not considered and thus are subject to refinement.

Future work should account for photochemical changes to optical (e.g., absorbance) and physical (e.g., $\mathrm{O}_{2}$ diffusion rates) properties of PS with increased time in the environment, differences in reactivity across product formulations (e.g., wavelength dependence), residence times of PS in sunlit environments, and the lability of partial photo-oxidation products to microbial respiration.

\subsection{Radiocarbon and Stable Carbon Isotope Conservative Tracer Experiment}


We used natural abundance radiocarbon as a conservative tracer of PS complete photooxidation to $\mathrm{CO}_{2}$. Strips of Goodfellow PS (1.5 x 7") were placed into $600-\mathrm{mL}$ quartz Erlenmeyer flasks $(\mathrm{N}=6)$. The flasks were filled with laboratory atmosphere equilibrated synthetic seawater (prepared as described above in section 1.3) and capped with groundglass $24 / 40$ stoppers (no headspace). All glassware was pre-combusted. Three vials were wrapped in aluminum foil and submerged in a water bath set at $20^{\circ} \mathrm{C}$. The other three vials were submerged in a water bath set at $20^{\circ} \mathrm{C}$ and exposed to seven days of simulated sunlight, an exposure time chosen to detect appreciable differences in DIC concentration and isotopic composition, relative to the dark-controls. Following irradiation, the samples were submitted to the National Ocean Sciences Accelerator Mass Spectrometry facility (NOSAMS) for the concentration and $\Delta^{14} \mathrm{C}$ and $\delta^{13} \mathrm{C}$ values of the DIC. One dark-control was lost on the DIC extraction line. The isotopic composition of the parent PS was also analyzed at NOSAMS using elemental analysis. The results of this experiment are presented in Figure 1C and Table S4. 
Predicted DIC $\Delta^{14} \mathrm{C}$ values for the light-exposed treatments were determined using a $\Delta^{14} \mathrm{C}$ mass balance:

$\Delta^{14}$ DIC $C_{\text {Final }}=f_{\text {DIC,Dark }} \Delta^{14}$ DIC $C_{\text {Dark }}+\left(1-f_{\text {DIC,Dark }}\right) \Delta^{14} C_{P S}$

where $\Delta^{14} \mathrm{DIC}$ Final is the measured $\Delta^{14} \mathrm{C}$ content of the DIC after irradiation via AMS, $\Delta^{14} \mathrm{DIC}_{\text {Dark }}$ is the measured $\Delta{ }^{14} \mathrm{C}$ content of the DIC in the dark-controls, and $\Delta^{14} \mathrm{C}_{\mathrm{PS}}$ is the measured $\Delta^{14} \mathrm{C}$ content of Goodfellow PS. The measured mass fraction of DIC in the dark-controls is represented as $f_{D / C, D a r k}$, whereas $\left(1-f_{D / C, D a r k}\right)$ is the mass fraction of the photochemically produced DIC (measured).

A similar mass balance was used to calculate the kinetic isotope effect of $\delta^{13} \mathrm{C}$ during complete photo-oxidation of PS $\left(\varepsilon_{P S}\right)$ :

$\delta^{13}$ DIC Final $=f_{\text {DIC,Dark }} \delta^{13}$ DIC Dark $+\left(1-f_{\text {DIC,Dark }}\right)\left(\delta^{13} C_{P S}+\varepsilon_{P S}\right)$

\subsection{Wavelength Dependence}

Given that Trycite 8001 has no detectable absorbance in the visible region and Trycite 8003 has appreciable visible light absorbance, we tested the hypothesis that Trycite 8001 
is not photochemically reactive and Trycite 8003 is photochemically reactive in the visible region. Films of Trycite 8001 and $8003(1.5 \times 3 \mathrm{~cm})$ were placed into air-tight 3-mL borosilicate exetainers (LabCo Inc.). The exetainers were filled with synthetic seawater with no headspace, placed into the sample chamber, and exposed for 8 hours to $450 \pm$ $26 \mathrm{~nm}$ light ( \pm full width at half-maximum; Fig. 2B). The light source is a Xe-KiloArc system equipped with a monochromator for waveband tuning (custom designed by Horiba Scientific Inc.). A water-cooled aluminum block held the sample vial and maintained temperature at $22^{\circ} \mathrm{C}$ throughout the exposure. Dark-controls were wrapped in aluminum

foil and maintained at $22^{\circ} \mathrm{C}$. All samples were analyzed for their dissolved oxygen content using membrane inlet mass spectrometry. The results are presented in Figure $2 \mathrm{C}$ of the main text.

\subsection{Elemental Analysis}

All PS sources were analyzed in triplicate by Midwest Laboratories for their carbon, hydrogen, nitrogen, oxygen, and sulfur content using elemental analysis. The results are presented in Table S3. 

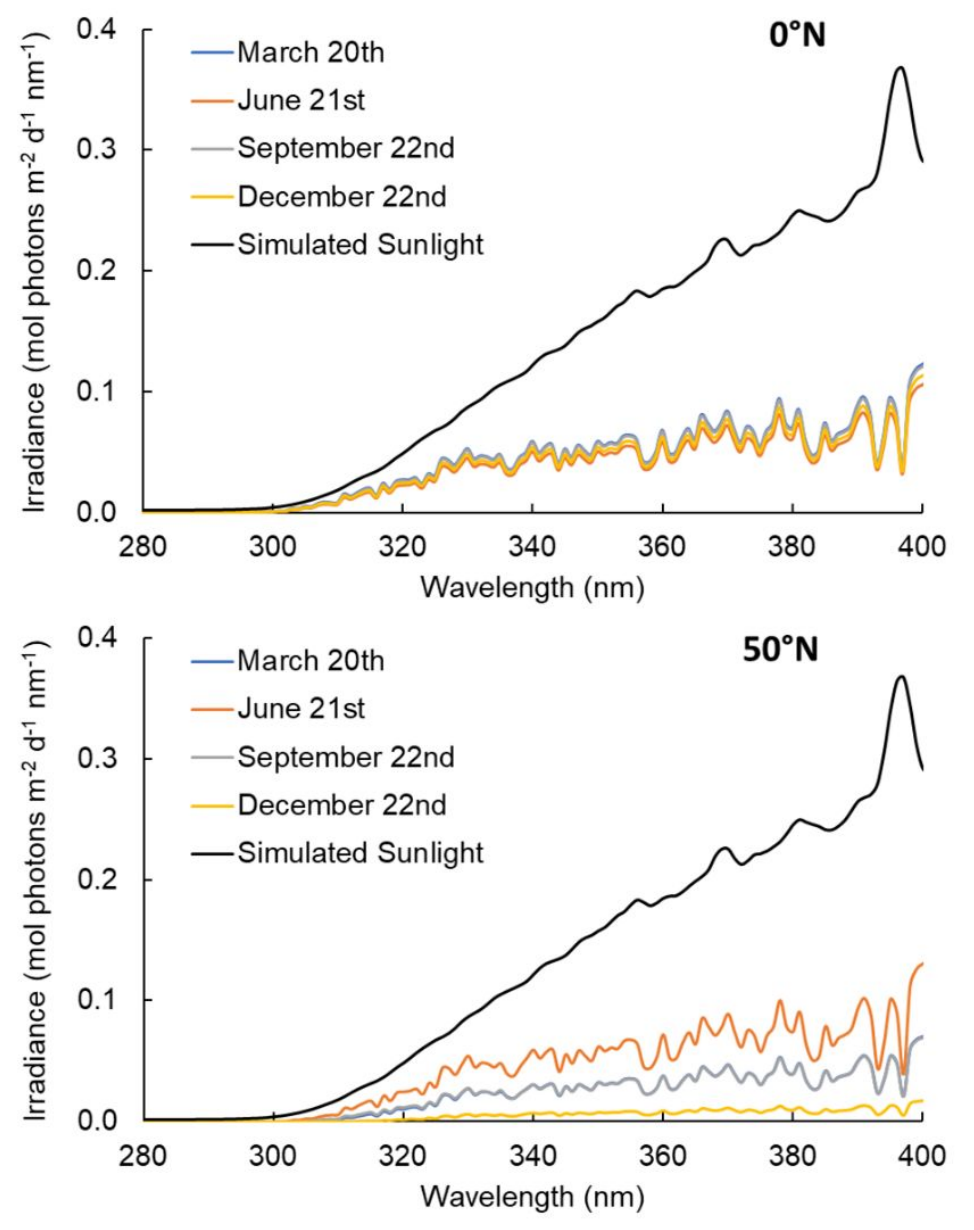

Figure S1. Comparison of simulated sunlight used in this study to natural sunlight at 0 and $50^{\circ} \mathrm{N}$ in all four seasons. Natural sunlight reference spectra were previously reported. ${ }^{6}$ 


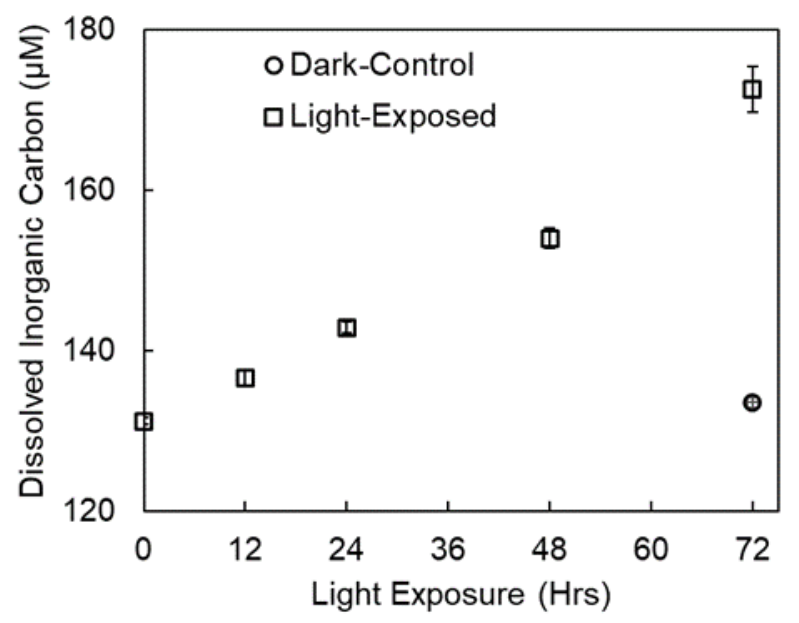

Figure S2. Complete photo-oxidation of PS to dissolved inorganic carbon increases with increasing exposure to simulated sunlight. Error bars represent standard error from the mean $(\mathrm{N}=4)$. 
Table S1. Photochemical oxygen consumption and carbon dioxide production of five polystyrene sources. Error bars represent one standard error from the mean $(\mathrm{N}=3$ for thickness, $\mathrm{N}=4$ for $\mathrm{O}_{2}$ consumption and $\mathrm{CO}_{2}$ production). 


\begin{tabular}{|c|c|c|c|c|}
\hline \multirow[t]{2}{*}{ PS Source } & Film Thickness & $\begin{array}{c}\text { Simulated Sunlight } \\
\text { Exposure }\end{array}$ & $\begin{array}{l}\text { Photochemical } \\
\mathrm{O}_{2} \text { Consumption }\end{array}$ & $\begin{array}{l}\text { Photochemical } \\
\mathrm{CO}_{2} \text { Production }\end{array}$ \\
\hline & $\mu m$ & hours & $\mu M$ & $\mu M$ \\
\hline Goodfellow & $192 \pm 1$ & 9 & $79 \pm 1$ & $19 \pm 4$ \\
\hline Sigma $35 \mathrm{~K}$ & $\mathrm{n} / \mathrm{a}$ & 12 & $113 \pm 2$ & $9 \pm 1$ \\
\hline Sigma 192K & $\mathrm{n} / \mathrm{a}$ & 6 & $107 \pm 2$ & $59 \pm 8$ \\
\hline Trycite 8001 & $44 \pm 1$ & 48 & $82 \pm 4$ & $15 \pm 4$ \\
\hline Trycite 8003 & $56 \pm 1$ & 12 & $74 \pm 4$ & $4 \pm<1$ \\
\hline
\end{tabular}


Table S2. Ratio of simulated to natural irradiance, integrated from $280-400 \mathrm{~nm}$. Natural sunlight reference spectra were previously reported. ${ }^{6}$

\begin{tabular}{|c|c|c|c|c|c|c|}
\hline \multicolumn{2}{|c|}{$\begin{array}{c}\text { Simulated to Natural } \\
\text { Irradiance }(280-400 \mathrm{~nm})\end{array}$} & \multicolumn{6}{|c|}{ Season } \\
\cline { 2 - 7 } & $0^{\circ} \mathrm{N}$ & 3 & 4 & 3 & 3 & 3 \\
\cline { 2 - 7 } & $10^{\circ} \mathrm{N}$ & 3 & 3 & 3 & 4 & 3 \\
\cline { 2 - 7 } & $20^{\circ} \mathrm{N}$ & 3 & 3 & 3 & 5 & 4 \\
\cline { 2 - 7 } & $30^{\circ} \mathrm{N}$ & 4 & 3 & 4 & 7 & 4 \\
\cline { 2 - 7 } & $40^{\circ} \mathrm{N}$ & 4 & 3 & 4 & 11 & 6 \\
\cline { 2 - 7 } & $50^{\circ} \mathrm{N}$ & 6 & 3 & 6 & 24 & 10 \\
\hline
\end{tabular}


Table S3. Elemental composition of five polystyrene sources. Error bars represent one standard error from the mean of triplicates. $n d=$ not detectable.

\begin{tabular}{|l|l|l|l|l|l|}
\hline \multicolumn{1}{|c|}{$\%$} & Goodfellow & Sigma 35 K & Sigma 192 K & Trycite 8001 & Trycite 8003 \\
\hline Carbon & $91.5 \pm<0.1$ & $91.6 \pm 0.1$ & $91.8 \pm 0.2$ & $91.4 \pm<0.1$ & $91.3 \pm 0.1$ \\
\hline Hydrogen & $8.2 \pm 0.1$ & $7.9 \pm 0.1$ & $7.9 \pm 0.1$ & $8.3 \pm<0.1$ & $8.3 \pm 0.1$ \\
\hline Oxygen & nd & nd & nd & nd & nd \\
\hline Nitrogen & nd & nd & nd & nd & nd \\
\hline Sulfur & $0.2 \pm<0.1$ & $0.3 \pm<0.1$ & $0.4 \pm<0.1$ & $0.3 \pm<0.1$ & $0.6 \pm 0.1$ \\
\hline
\end{tabular}


Table S4. Results from the isotope conservative tracer experiment described in section 1.5. Error bars represent one standard error from the mean. $\mathrm{n} / \mathrm{a}=$ not applicable. 


\begin{tabular}{|l|c|c|c|}
\hline \multicolumn{1}{|c|}{ Treatment } & {$[\mathrm{DIC}](\mu \mathrm{M})$} & $\Delta^{14} \mathrm{C}(\%)$ & $\delta^{13} \mathrm{C}(\%)$ \\
\hline Polysyrene $(\mathrm{N}=3)$ & $\mathrm{n} / \mathrm{a}$ & $-1000 \pm<1.0$ & $-30.3 \pm<0.1$ \\
\hline Dark-Control $(\mathrm{N}=2)$ & $35 \pm 1$ & $-202 \pm 8$ & $-12.6 \pm<0.1$ \\
\hline Light-Exposed $(\mathrm{N}=3)$ & $46 \pm 1$ & $-381 \pm 5$ & $-19.2 \pm 0.1$ \\
\hline
\end{tabular}


Table S5. First-order rates of complete (PM) and partial (PPO) photo-oxidation in the solar simulator. Using the scaling factors from Table S2, these rates were used to estimate environmental half-lives in years (see Section 1.4 for details).

\begin{tabular}{|c|c|c|c|c|c|c|}
\hline & \multicolumn{6}{|c|}{ First-Order Degradation Rate in Solar Simualtor $\left(\mathrm{k} ; \mathrm{hr}^{-1}\right)$} \\
\hline & \multicolumn{3}{|c|}{ Goodfellow } & \multicolumn{3}{|c|}{ Trycite 8003} \\
\hline & PM & PPO & $\mathrm{PM}+\mathrm{PPO}$ & PM & $\mathrm{PPO}$ & $P M+P P O$ \\
\hline Average & $1.3 \mathrm{E}-06$ & $8.3 \mathrm{E}-06$ & $9.7 E-06$ & $9.0 \mathrm{E}-07$ & $2.8 \mathrm{E}-05$ & $2.9 \mathrm{E}-05$ \\
\hline \multirow[t]{3}{*}{ SD } & $5.7 \mathrm{E}-07$ & $1.2 \mathrm{E}-06$ & $6.5 \mathrm{E}-07$ & $1.8 \mathrm{E}-07$ & $4.6 \mathrm{E}-06$ & $4.8 \mathrm{E}-06$ \\
\hline & \multicolumn{6}{|c|}{ Average Environmental Half-Life (Years) } \\
\hline & \multicolumn{3}{|c|}{\begin{tabular}{l|l} 
Goodfellow \\
\end{tabular}} & \multicolumn{3}{|c|}{ Trycite 8003} \\
\hline Latitude & PM & PPO & $\mathrm{PM}+\mathrm{PPO}$ & PM & PPO & $P M+P P O$ \\
\hline $0^{\circ} \mathrm{N}$ & 190 & 30 & 30 & 290 & 10 & 10 \\
\hline $10^{\circ} \mathrm{N}$ & 200 & 30 & 30 & 290 & 10 & 10 \\
\hline $20^{\circ} \mathrm{N}$ & 220 & 40 & 30 & 320 & 10 & 10 \\
\hline $30^{\circ} \mathrm{N}$ & 260 & 40 & 40 & 380 & 10 & 10 \\
\hline $40^{\circ} \mathrm{N}$ & 340 & 60 & 50 & 510 & 20 & 20 \\
\hline $50^{\circ} \mathrm{N}$ & 570 & 90 & 80 & 840 & 30 & 30 \\
\hline Average & 300 & 50 & 40 & 440 & 20 & 20 \\
\hline SD & 150 & 20 & 20 & 210 & 10 & 10 \\
\hline
\end{tabular}




\section{References:}

(1) Gewert, B.; Plassmann, M.; Sandblom, O.; MacLeod, M. Identification of Chain Scission Products Released to Water by Plastic Exposed to Ultraviolet Light. Environ. Sci. Technol. Lett. 2018, 5, 272-276.

(2) Ward, C. P.; Sharpless, C. P.; Valentine, D. L.; French-McCay, D.; Aeppli, C.; White, H. K.; Rodgers, R. P.; Gosselin, K. M.; Nelson, R. K.; Reddy, C. M. Partial Photochemical Oxidation Was a Dominant Fate of Deepwater Horizon Surface Oil. Environ. Sci. Technol. 2018, 52, 1797-1805.

(3) Ward, C. P.; Sleighter, R. L.; Hatcher, P. G.; Cory, R. M. Insights into the Complete and Partial Photooxidation of Black Carbon in Surface Waters. Environ. Sci. Process. Impacts 2014, 16, 721-731.

(4) Cory, R. M.; Ward, C. P.; Crump, B. C.; Kling, G. W. Sunlight Controls Water 
Column Processing of Carbon in Arctic Fresh Waters. Science 2014, 345, 925-928.

(5) Ward, C. P.; Cory, R. M. Chemical Composition of Dissolved Organic Matter Draining Permafrost Soils. Geochim. Cosmochim. Acta 2015, 167, 63-79.

(6) Apell, J. N.; McNeill, K. Updated and Validated Solar Irradiance Reference Spectra for Estimating Environmental Photodegradation Rates. Environ. Sci. Process. Impacts 2019, 21, 427-437.

(7) Schmidt, C.; Krauth, T. Export of Plastic Debris by Rivers. Environ. Sci. Technol. 2017, 51, 12246-12253.

(8) Gewert, B.; Plassmann, M. M.; MacLeod, M. Pathways for Degradation of Plastic Polymers Floating in the Marine Environment. Environ. Sci. Process. Impacts 2015, 17, 1513-1521.

(9) Khaled, A.; Rivaton, A.; Richard, C.; Jaber, F.; Sleiman, M. Phototransformation of Plastic Containing Brominated Flame Retardants: Enhanced Fragmentation and Release of Photoproducts to Water and Air. Environ. Sci. Technol. 2018, 52, 11123-11131. 